\title{
Structural Equation Modeling of the Relationship of Bone Mineral Density and Its Risk Factors in Japanese Women
}

\author{
Satoshi TOYOKAWA ${ }^{1}$, Hiroaki NISHIKAWA², Masaru UEJI ${ }^{3}$, \\ Kazuo MOTEGI ${ }^{4}$ and Katsumi KANO ${ }^{3}$ \\ ${ }^{1}$ Graduate School of Medicine, University of Tsukuba, Ibaraki \\ ${ }^{2}$ College of Medical Technology \& Nursing, University of Tsukuba, Ibaraki \\ ${ }^{3}$ Institute of Community Medicine, University of Tsukuba, Ibaraki \\ ${ }^{4}$ Tsukuba Health Service Center, Ibaraki
}

\begin{abstract}
Several factors have been reported as risk factors for the development of osteoporosis. In this study, we aimed to examine the relationship among lifestyle factors, biologic factors, and bone mineral density (BMD) using structural equation modeling (SEM). The subjects in the present study consisted of 866 postmenopausal Japanese women aged between 40 and 80 years old. In the analysis by the SEM, we employed a multiple basic model. As the structural variables, lifestyle factors and biologic factors were selected.

The goodness of fit index (GFI) of the final model was 0.991 and the Akaike's information criteria (AIC) showed the lowest value in the peripheral models. The degree of association between biologic factors and BMD was $-\mathbf{0 . 5 7 6}$ (direct association), 0.012 (indirect association), and -0.564 (total association). With regard to the correlation between lifestyle factors and BMD, the degrees of association were $\mathbf{0 . 0 8 5}, \mathbf{- 0 . 0 8 4}$, and 0.001 , respectively.

This study defined a pilot model for factors influencing BMD. Although is remains necessary to conduct further analyses with more valid measurements and constructs, this model indicated that the correlation between BMD and lifestyle factors was lower than that between BMD and biologic factors.
\end{abstract}

Key words: osteoporosis, bone mineral density, lifestyle, structural equation modeling

\section{Introduction}

Osteoporosis is a serious public health issue and one of the most important disorders associated with aging ${ }^{1)}$. The number of patients with osteoporosis among women aged 50 years and over was estimated to be between 5 million and 8.5 million in Japan ${ }^{2)}$, whereas it was assumed to be approximately 4-6 million in the $\mathrm{US}^{3)}$. The direct and indirect medical costs of osteoporosis, which were estimated as $\$ 6.1$ billion in the United States, have become a severe social problem ${ }^{2,3)}$. Meanwhile, it has been reported that osteoporosis predisposes sufferers to fractures, which can cause the elderly to become bedridden, to degrade their quality of life $\mathrm{e}^{4-8}$ and increases the risk of death ${ }^{9}$. Accordingly, osteoporosis has significant medical, social and economic implications ${ }^{10,11)}$, and has been a subject of numerous studies.

Risk factors for the development of osteoporosis have been reported such as lack of sufficient physical activity, low intake of

Received Nov. 2, 2000/Accepted Jan. 25, 2001

Reprint requests to: Satoshi TOYOKAWA

Graduate School of Medicine, University of Tsukuba, Tennoudai 1-1-1,

Tsukuba, Ibaraki 305-8575, Japan

TEL \& FAX: +81(298)53-3117 calcium and vitamin D, low level of exposure to sunlight, smoking habit, weight, obesity, estrogen supplementation therapy, early menopause, hyperthyroidism and diabetes mellitus ${ }^{1,12-15}$. One of the objectives of the present study was to clarify the relationship between each risk factor and bone mineral density (BMD), structurally. In particular, to indicate the relationship between BMD and modifiable lifestyle factors, and that between BMD and biologic factors, it is important to examine the efficacy of prevention by improvement of lifestyle. Since lifestyle is an abstract concept, it should be treated as a "construct". Constructs are concepts, which are applied to simplify the understanding of relatively complex phenomena by hypothesizing its existence ${ }^{16)}$. Structural equation modeling (SEM), which can introduce constructs into the analysis, allows examination of the relationships among the characteristics of the constructs, other constructs and the observed variables $^{16-18)}$. In the present study, we aimed to demonstrate the relationships among modifiable lifestyle factors, biologic factors, and BMD with SEM.

\section{Materials and Methods}

Subjects

The study was conducted between July 1996, and March, 
1997 in Ibaraki Prefecture, Japan through the Prefectural Osteoporosis Screening Program. The program was conducted for the early detection and treatment of osteoporosis in 14 public health centers in Ibaraki Prefecture. The study subjects were 1,236 postmenopausal Japanese women between 40 and 80 years old, who voluntarily participated in the screening and the study. Among the 1,236 Japanese women, those with diseases or operations known as risk factors of secondary osteoporosis such as thyroid disease $(n=75)$, parathyroid disease $(n=3)$, diabetes mellitus $(n=36)$, renal disease $(n=37)$, gastroenterotomy $(n=30)$ and ovariectomy $(n=155)$, or the use of oral contraceptives $(n=6)$, were excluded from the analysis to control confounding factors. Forty-three women with a history of smoking were also excluded from the analysis to control its confounding effects. Finally, 866 women were included in the analysis. All subjects were clearly informed about the screening process. Written informed consent was obtained from all subjects participating in the study.

\section{Measurements}

We conducted a self-administered questionnaire survey to obtain information on the present and past medical history, physical activity and diet pattern, and reproductive history of the subjects simultaneously with the screening. The questionnaire contained questions on average frequency of physical activity per week, which was continued to the present and performed in the past. To obtain information on the subjects' diet, ten items related to dietary balance, six items related to calcium intake and six items related to unwholesome diets were recorded. Table 1 shows the questions about dietary balance, calcium intake, and unwholesome diet. For the scores for dietary balance and unwholesome diets, we employed a two-point scale, rating a satisfied response as 1, and dissatisfied response as 0 . For the score about calcium intake, we

Table 1 Questionnaire for dietary balance, calcium intake, and unwholesome diet

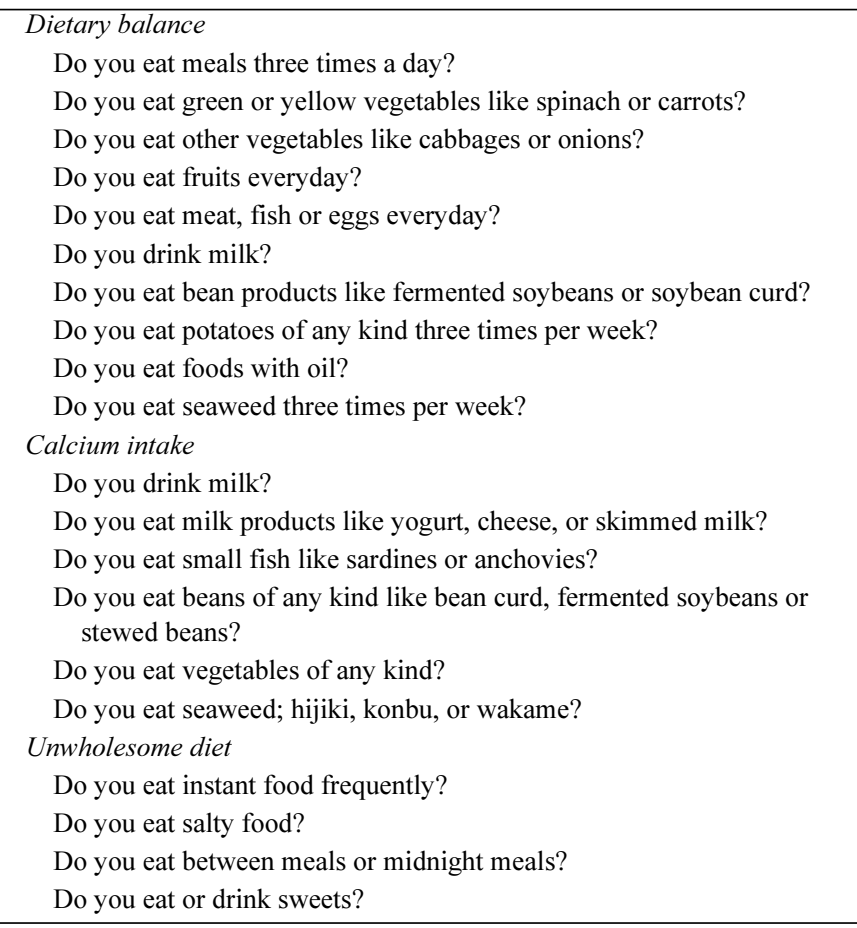

Answers for Dietary balance and Unwholesome diet were 'yes' or 'no'. Answers for Calcium intake were 'three times or more per week', 'one or two times per week' or 'seldom'. employed a three-point scale, rating 'three times or over a week' as 2, 'one or two times a week' as 1 , and a 'seldom' as 0 . To obtain information on physical activity, we asked about average frequency of current and past physical activity per week. To obtain information on the reproductive history, the age of menarche and menopause, and the number of childbirths were recorded. Subjects answered the questionnaire with the guidance by public health nurses. The examiners checked each questionnaire to ensure that the subjects had answered all the questions. Height and weight were measured at physical check-up. Grip strength in both hands was measured with a hand-held squeeze dynamometer. Three grip strength measurements were made for each hand, and the mean of the three measurements was recorded.

BMD was measured from a radiograph of the second metacarpal of the right hand by computed X-ray densitometry using a bonalyzer (Teijin Ltd., Tokyo, Japan). In this method, BMD was calculated by comparing the optical density of aluminum step wedges as the standard and the X-ray absorption density of the bone from the radiograph ${ }^{19-21)}$. This measurement of BMD from a radiograph of the hand was not influenced by the soft tissue density and is a highly reproducible and simple operation. Two densitometers were used at all the screening facilities. Reliable calibration and stability of both densitometers were ensured by daily phantom measurements. The coefficient of variation (CV) for repeated measures (same technician examined the same X-ray film phantom 6 times) and daily (same technician examined the same X-ray film phantom for 9 days) was $0.70 \%$ and $1.45 \%$. The inter-assessors reliability was $1.04 \%(\mathrm{CV})$. To examine the in vivo reliability, 15 normal subjects were analyzed: The in vivo precision was $1.31 \%(\mathrm{CV})$. Detailed information about the method of BMD measurement has been documented previously ${ }^{22,23)}$.

\section{Data analysis}

In the analysis by SEM, we employed a multiple basic model in which the observed variables were used as indices for each of the structural variables, and the relationships among the structural variables were studied. In the basic model, biologic factors and lifestyle factors were selected as the structural variables, which were related to BMD directly. Biologic factors consisted of age, height, weight, body mass index (BMI), grip strength of the dominant hand, age of menarche and menopause, number of years between menarche and menopause, numbers of years from menopause to the present, and the frequency of pregnancies and childbirths as observed variables in the basic model. Lifestyle factors consisted of frequency of past and present physical activity, scores of calcium intake, dietary balance and unwholesome diet as observed variables in the basic model.

Among all the models, which could be structured in combinations with the aforementioned factors, the final model was determined using the goodness of fit index (GFI) and Akaike's information criteria (AIC). Among the models with 0.99 or higher GFI, that which had the lowest AIC was selected as the final model. GFI is a proportion in which the covariance matrix of the structured model explains a sample covariance matrix and is also an index suitable for relative model evaluation. AIC which has a significantly superior model selection function than GFI is considered suitable for relative model evaluation ${ }^{16-18)}$. To examine the final model, we calculated the direct association, indirect association and total association of the variables in the final model. Direct association refers to the strength of association through only one 
Table 2 Mean values and standard deviations for each observed variable

\begin{tabular}{lrr}
\hline Variables & $\mathrm{N}$ & Mean $\pm \mathrm{SD}$ \\
\hline Biologic factors & & \\
Age (years) & 866 & $59.52 \pm 6.69$ \\
Height $(\mathrm{cm})$ & 866 & $151.12 \pm 5.46$ \\
Weight $(\mathrm{kg})$ & 866 & $53.69 \pm 7.49$ \\
Body mass index $\left(\mathrm{kg} / \mathrm{m}^{2}\right)$ & 866 & $23.51 \pm 3.07$ \\
Grip strength $(\mathrm{kg})$ & 866 & $23.48 \pm 4.82$ \\
Age at menarche (years) & 866 & $15.12 \pm 2.75$ \\
Age at menopause (years) & 866 & $49.27 \pm 4.29$ \\
Childbirth & 861 & $2.29 \pm 1.03$ \\
Reproductive period (years) & 865 & $34.15 \pm 5.12$ \\
Post menopause period (years) & 866 & $10.25 \pm 7.32$ \\
Lifestyle factors & & \\
Calcium Intake & 866 & $7.92 \pm 1.72$ \\
Dietary balance & 866 & $4.76 \pm 2.20$ \\
Unwholesome diet & 866 & $2.04 \pm 1.03$ \\
Current physical activity & 856 & $1.00 \pm 2.01$ \\
Past physical activity & 855 & $1.26 \pm 1.71$ \\
Dependent variable & & \\
Bone mineral density ( $\Sigma \mathrm{GS} / \mathrm{D})$ & 866 & $2.39 \pm 0.29$ \\
\hline
\end{tabular}

SD: Standard deviation

structural variable, and indirect association refers to the strength of association through two structural variables in this modeling. Both direct and indirect associations were calculated by multiplying the partial standardized regression coefficients derived from each variable. The total association is a sum of the direct and indirect associations. The CALIS procedure of SAS, a statistical software package, was used for the SEM ${ }^{18)}$.

\section{Results}

Table 2 shows the numbers of subjects, mean values, and standard deviation of the observed values. The mean age and BMD were 59.3 (standard deviation (SD): 6.7) and 2.39 (SD: 0.3), respectively.

Table 3 Adequacy of the model to fit for the final model and its peripheral models

\begin{tabular}{lccc}
\hline Observed variables $^{* 1}$ & GFI $^{* 2}$ & AGFI $^{* 3}$ & AIC $^{* 4}$ \\
\hline Peripheral model & & & \\
A, PP, AM, CB, CI, DB, UD, PPA, CPA & 0.993 & 0.988 & -23.384 \\
A, PP, AM, CB, CI, DB, UD, $\quad 0.988$ & -23.174 \\
A, PP, AM, CB, CI, DB, PPA, CPA & 0.993 & 0.987 & -22.350 \\
A, PP, AM, CB, CI, UD, PPA, CPA & $\#$ & & \\
A, PP, AM, CB, DB, UD, PPA, CPA & $\#$ & & -18.400 \\
A, PP, AM, CI, DB, UD, PPA, CPA & 0.992 & 0.985 & -18.341 \\
A, PP, CB, CI, DB, UD, PPA, CPA & 0.992 & 0.985 & \\
A, AM, CB, CI, DB, UD, PPA, CPA & $\#$ & & \\
PP, AM, CB, CI, DB, UD, PPA, CPA & $\#$ & & \\
Final model & & & \\
A, PP, AM, CB, CI, DB, UD, PPA, CP & 0.991 & 0.986 & -28.680 \\
\hline *1 A: Age, PP: Postmenopausal period, AM: Age at menarche, CB: Child- & \\
birth, CI: Calcium intake, DB: Dietary balance, UD: Unwholesome diet, \\
PPA: Past physical activity, CPA: Current physical activity
\end{tabular}

Table 4 Association between each variable and bone mineral density according to the structural equation model in Japanese elderly women

\begin{tabular}{lrrr}
\hline Variables & $\begin{array}{c}\text { Direct } \\
\text { association }\end{array}$ & $\begin{array}{c}\text { Indirect } \\
\text { association }\end{array}$ & $\begin{array}{c}\text { Total } \\
\text { association }\end{array}$ \\
\hline Latent variables & -0.576 & 0.012 & -0.564 \\
Biologic factors & 0.085 & -0.084 & 0.001 \\
Lifestyle factors & & & \\
Observed variables & -0.566 & 0.012 & -0.554 \\
Age & -0.479 & 0.010 & -0.469 \\
Postmenopausal period & -0.100 & 0.002 & -0.098 \\
Age at menarche & -0.099 & 0.002 & -0.097 \\
Childbirth & 0.052 & -0.051 & 0.001 \\
Calcium intake & 0.067 & -0.066 & 0.001 \\
Dietary balance & -0.004 & 0.004 & 0.000 \\
Unwholesome diet & 0.007 & -0.007 & 0.000 \\
Past physical activity & 0.014 & -0.014 & 0.000 \\
Current physical activity & & & \\
\hline
\end{tabular}

Table 3 shows the goodness of fit of the final model and its peripheral model. The GFI and AIC of the final model were 0.991 and -28.680 , respectively. Figure 1 shows a path diagram of the final model. The causal correlation coefficient for each variable is indicated above the arrow. In the final model, lifestyle factors consisted of calcium intake, dietary balance, past physical activity, and current physical activity positively and unwholesome diet negatively. Passive factors consisted of age, postmenopausal period, age at menarche, and childbirth positively. The direct associations between lifestyle factors and BMD $(0.085)$ was weaker than the one between biologic factors and BMD $(-0.576)$.

Table 4 shows direct, indirect, and total association of the variables and BMD in the final model. The total association between biologic factors and BMD $(-0.564)$, the sum of its direct association and indirect association (0.012). It was bigger than the total association between lifestyle factors and BMD (0.001) which was the sum of its direct association and indirect association $(-0.084)$.

With regard to the correlation between each observed variable and BMD, the biggest degrees of association were for current age; direct association: -0.566 , indirect association: 0.012 , and total association: -0.554 . Associations between each observed variables in biologic factors and BMD were greater than those among the observed variables in lifestyle factors and BMD.

\section{Discussion}

The strongest correlation in the final model was observed between age and BMD of all the correlations between each observed variable and BMD. This result was consistent with previous report, which clarified negative associations between age and $\mathrm{BMD}^{14,24,26)}$. In the final model, postmenopausal years, age at menopause and frequency of childbirths were significantly correlated with BMD. These reproductive factors were assumed to be correlated with estrogen exposure, which has a significant influence on bone metabolism ${ }^{1,13,22,27)}$. Many studies have confirmed a positive correlation between BMD and each observed variable of physical composition; height, weight, obesity and grip strength. Regarding the preventive effect of weight it is suggested that physical burden due to weight stimulated an increased $\mathrm{BMD}^{28-31}$. Meanwhile, it was assumed that secretion of estrogen from mast 


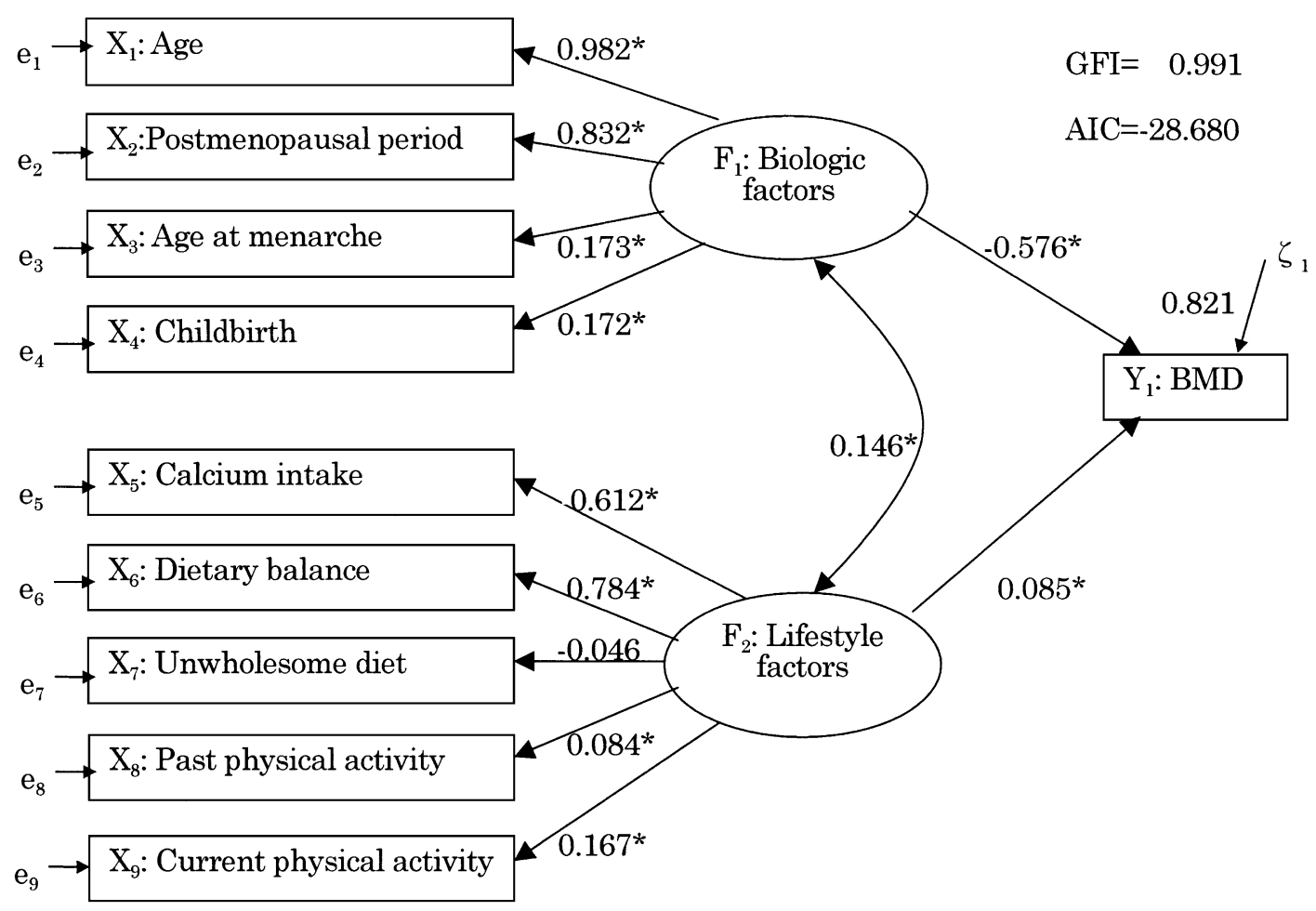

Fig. 1 Path diagram from the final model, showing intake factors and freestanding variables on the left and outcome factors on the right. Numbers on lines represent partial standardized regression coefficients, representing the unique contribution of the predictors to the outcome. Circles represent latent variables. Boxes represent observed variables. Straight arrows represent effects. Curved arrow represents covariance. $* \mathbf{p}<\mathbf{0 . 0 5}$

cells also contributed to the preventive effect of osteoporosis with obesity $^{30)}$. Although a positive correlation between grip strength and BMD has also been reported ${ }^{23,32)}$, this correlation was not consistent with the final model of the present study. In the current analysis, the models that included weight, BMI, or grip strength, were discarded since the indicators for goodness of fit for the models were low.

According to lifestyle factors, the present findings suggested that BMD would be more strongly correlated with calcium intake and dietary balance than with frequency of physical activity. Some studies have compared diet and physical activity to study the effects of prevention against osteoporosis; however, their observations were not consistent ${ }^{33-36)}$. In previous studies, the efficacy of prevention through both diet and physical activity has been indicated ${ }^{19,33-35)}$. Both dietary factors and physical activity factors were included in the final model, which indicated their combined efficacy in the prevention of osteoporosis.

The final model could be seen as a model to indicate that there were weak correlations between BMD and each observed variable for lifestyle, and that there was a weak correlation between BMD and physical composition; weight, BMI, and grip strength. These findings were inconsistent with the current consensus about the preventive effect of lifestyle ${ }^{19,27,33,36)}$. The accuracy and the validity of both the indicators and the constructs were not verified by other studies. It was likely that the low accuracy made the correlations between lifestyles and BMD weaker. Another reason to weaken the correlations was that some important factors were not included in the model such as smoking and drinking.

Constructs with SEM have been employed in mental health research because they are available for analyses of abstract ideas ${ }^{17}$. For control of osteoporosis, it was not sufficient only to study associations between the disease and each individual lifestyle as previous epidemiological studies have done, because it is also necessary to examine associations between the disease and the total lifestyle. For the latter examination further studies will be indispensable. SEM is applicable not only to analyze constructs but also to refine a model to fit with accumulated knowledge. A pilot model is necessary to develop a new and more precise model. The current finding proposed one pilot model for BMD and lifestyle factors.

The GFI and AIC of the final model were 0.991 and -28.680 , respectively, which indicated that the model fitted the data well. However the enough model did not explain associations among BMD and risk factors, since some important risk factors were excluded from the model. This was simply because model selection in the present study depended on the statistical indices for model fitting.

One limitation of this study was in its inability to clarify the efficacy of prevention because it was a cross-sectional model. To address this limitation, further longitudinal studies will be necessary. The present study recruited subjects who participated in the health examination for diagnosis of osteoporosis voluntarily. The voluntary participation might cause selection bias, which attenuated the generalizability of the study. This study focused on biologic factors and modifiable lifestyle factors and defined a multiple index model as a basic model. However, a different final model could be selected, if the viewpoints for the way of modeling, the indicators employed in the analysis and the subjects were different. To confirm the stability of the model, we calculated the goodness of fit for all 1,236 patients, including the 370 who were excluded from the study due to history of disease, operation or 
smoking. The model from this extra analysis showed a similar structural association and indicated a high goodness of fit with a GFI of 0.992 and AIC of -30.555 . This finding alone did not confirm the stability of the model, but it could be suggested that the final model was stable.

In conclusion, this study defined a model for the study of factors influencing BMD. Although it is still necessary to conduct further analyses with more valid measurements and constructs, the finding of the present study indicated that the correlation between BMD and lifestyle factors structured with calcium intake, dietary balance, unwholesome diet and current physical activity was weaker than that between BMD and biologic factors structured with age, postmenopausal period, age at menarche, and childbirth.

\section{Acknowledgments}

The authors express their deep gratitude to the members of public health centers, the department of welfare and the examination committee for the osteoporosis project in Ibaraki Prefecture.

\section{References}

1) Willhite L. Osteoporosis in woman: prevention and treatment. J. Am. Pharm. Assoc. 1998; 38: 614-624.

2) Suzuki T, Yoshida H, Ishizaki T. Epidemiology of osteoporosis: incidence, prevalence, and prognosis. Jpn. J. Clin. Med. 1998; 56: 1563-1568. (in Japanese)

3) Looker AC, Orwoll ES, Johnston CC Jr, Lindsay RL, Wahner HW, Dunn WL, et al. Prevalence of low femoral bone density in older U.S. adults from NHANES III. J. Bone Miner. Res. 1997; 12: 1761-1768.

4) Cook DJ, Guyatt GH, Adachi JD, Clifton J, Griffith LE, Epstein RS, et al. Quality of life issues in woman with vertebral fractures due to osteoporosis. Arthritis. Rheum. 1993; 36: 750-756.

5) Greendale GA, Silverman SL, Hays RD, Cooper D, Spector T, Kiel D, Reuben DB. Health-related quality of life in osteoporosis clinical trials. Calif. Tissue. Int. 1993; 53: 75-77.

6) Gold DT, Drenzner MK. Quality of life. In Riggs BL, Melton LJ (edrs). Osteoporosis: etiology, diagnosis, and management. 2nd ed. 1995, Philadelphia, Lippincott-Raven, 475-486.

7) Kanis JA, Minne WH, Meunier PJ, Ziegler R, Allender E. Quality of life and vertebral osteoporosis. Osteoporos. Int. 1992; 2: 161163.

8) Begerow B, Pfeifer M, Pospeschill M, Scholz M, Schlotthauer T, Lazarescu A, et al. Time since vertebral fracture: an important variable concerning quality of life in patients with postmenopausal osteoporosis. Osteoporos. Int. 1999; 10: 26-33.

9) Center JR, Nguyen TV, Schneider D, Sambrook PN, Eisman JA. Mortality after all major types of osteoporotic fracture in men and women: an observational study. Lancet 1999; 353: 878-882.

10) Lindsay R. The growing problem of osteoporosis. Osteoporos. Int. 1992; 2: 267-268.

11) Cummings SR, Rubin SM, Black $D$. The future of hip fractures in the United States. Numbers, costs, and potential effects of postmenopausal estrogen. Clin. Orthop. 1990; 252: 163-166.

12) Toyokawa S, Douglas Osei-Hyiaman, Ueji M, Takahashi H, Kano K. An epidemiological study of the relationship between bone mineral density and sever obesity. Igaku to Seibutsugaku 1997; 134: 217-221. (in Japanese)

13) Kanis JA, McCloskey EV. Risk factors in osteoporosis. Maturitas 1998; 30: 229-233.

14) Kanis JA. Osteoporosis: a view into the next century. Neth. J. Med. 1997; 50: 198-203.

15) Riggs BL, Melton LJ III. Involutional osteoporosis. N. Engl. J. Med. 1986; 314: 1676-1685.

16) Bollen KA. Structural equations with latent variables. John Wiley and Sons, New York, 1989.

17) Bollen KA, Long JS. Testing Structural equations Models. SAGE Publications, Newbury Park, 1993.

18) Toyoda H. Covariance Structure Analysis with SAS (Stastical

data analysis using SAS3). University of Tokyo press, Tokyo, 1992 (in Japanese).

19) Kano K. Relationship between exercise and bone mineral density between over 5,000 women aged 40 years and above. J. Epidemiol. 1998; 8: 28-32.

20) Matsumoto $C$, Kushida $K$, Yamazaki $K$, Imose $K$, Inoue $T$. Metacarpal bone mass in normal and osteoporotic Japanese women using computed X-ray densitometry. Calcif. Tissue Int. 1994; 55: 324-329.

21) Matsumoto C, Kushida K, Orimo H, Kosikawa Shouzou, Shiraki M, Akimoto T, et al. A new computed x-ray densitometor and its performance. J. Clin. Exp. Med. 1991; 156: 741-742. (in Japanese)

22) Osei-Hyiaman D, Toyokawa S, Ueji M, Takahashi H, Kano K. Timing of menopause, reproductive years, and bone mineral density. A cross-sectional study of postmenopausal Japanese Woman. Am. J. Epidemiol. 1998; 148: 1055-1061.

23) Osei-Hyiaman D, Ueji M, Toyokawa S, Takahashi H, Kano K. Influence of grip strength on metacarpal bone mineral density in postmenopausal Japanese woman: a cross-sectional study. Calcif. Tissue Int. 1999; 64: 263-266.

24) Melton LJ. Epidemiology of osteoporosis. Baillieres Clin. Obstet. Gynaecol. 1991; 5: 785-805.

25) Kanis JA, Melton LJ III, Christiansen C, Johnston CC, Khaltaev N. The diagnosis of osteoporosis. J. Bone Miner. Res. 1994; 9: 1137-1141.

26) Wei C, Yonemitsu H, Shibayama H, Ueda A. Investigation in Japanese using dual-energy x-ray absorptiometry. Jpn. J. Hyg. 1997; 51: 742-748. (in Japanese)

27) Sowers M. Pregnancy and lactation as risk factors for subsequent bone loss and osteoporosis. J. Bone Miner. Res. 1996; 11: 10521060 .

28) Edelstein SL, Barrett-Conner E. Relationship between body size and bone mineral density in elderly men and women. Am. J. Epidemiol. 1993; 138: 160-169.

29) Harris SS, Dawson-Hughes B. Weight, body composition, and bone density in postmenopausal women. Calcif. Tissue Int. 1996; 59: 428-432.

30) Ribot C, Tremollieres F, Pouilles JM. The effect of obesity on postmenopausal bone loss and the risk of osteoporosis. Adv. Nutr. Res. 1994; 9: 257-271.

31) Puente A, Postiglione A, Puente AE, Carpinelli A, Romano M, Oriente P. Peripheral body fat has a protective role on bone mineral density in elderly women. Eur. J. Clin. Nutr. 1998; 52: 690-693.

32) Preisinger E, Alacamioglu Y, Saradeth T, Resch KL, Gerold H, Metka M. Forearm bone density and grip strength in woman after menopause, with and without estrogen replacement therapy. 
Maturitas 1995; 21: 57-63.

33) Nelson M, Mayer AB, Rutherford O, Jones D. Calcium intake, physical activity and bone mass in pre-menopausal woman. J. Hum. Nutr. Diet. 1991; 4: 171-178.

34) Bauer DC, Browner WS, Cauley JA, Orwoll ES, Scott JC, Black $\mathrm{DM}$, et al. Factors associated with appendicular bone mass in older woman. Ann. Intern. Med. 1993; 118: 657-665.
35) Kano K, Haga M, Hosoda T, Suzuki M, Ueji M, Takahashi H, et al. A study of osteoporosis among females aged 50 years and over using mass screening data. Jpn. J. Health Hum. Ecol. 1996; 62: 186-196. (in Japanese)

36) Pritchard JE, Nowson CA, Wark JD. Bone loss accompanying diet-induced or exercise-induced weight loss: a randomized controlled study. Int. J. Obes. 1996; 20: 513-520. 